\title{
Relação entre potássio na solução do solo, umidade e condutividade elétrica aparente do solo ${ }^{1}$
}

\author{
Gessionei da S. Santana ${ }^{2}$, Eugênio F. Coelho ${ }^{3}$, Tibério S. M. da Silva ${ }^{3}$ \& Márcio M. Ramos ${ }^{4}$
}

\begin{abstract}
RESUMO
Através de modelos que relacionam umidade $(\theta)$, condutividade elétrica aparente do solo (CEa) e condutividade elétrica da solução do solo (CEw), objetivou-se avaliar, para condição de laboratório, a viabilidade de se estimar a concentração de potássio na solução do solo $(\mathrm{K})$, para solos de classe textural franca $\left(\mathrm{CT}_{\mathrm{f}}\right)$ e franco-arenosa $\left(\mathrm{CT}_{\mathrm{fa}}\right)$. Cinco soluções de cloreto de potássio, referentes a cinco condutividades elétricas $(1,0,2,5,4,0,5,5$ e 7,0 dS m-1), foram aplicadas sobre o solo acondicionado em colunas de PVC, de forma a se obter cinco $\theta$ do solo $(17,0 \pm 1,4,19,0 \pm 1,8,21,4 \pm 2,2,23,4 \pm 2,2$ e 26,0 $\pm 3,0 \%$, em volume). Efetuaram-se leituras de $\theta$ e CEa com um aparelho de reflectometria no domínio do tempo (TDR) e se extraiu solução do solo com extrator de cápsula de cerâmica, para determinar a CEw e o K. Três modelos foram ajustados aos dados, por meio de planilha eletrônica. É viável estimar a concentração de K na solução do solo, a partir de $\theta$ e CEa, para condição de laboratório, por meio dos modelos de Rhoades et al. (1976), Vogeler et al. (1996) e Mualen \& Friedman (1991), adaptados com uma relação entre CEw e $\mathrm{K}$ do tipo potência, nas faixas de 0 a 60 e 0 a $120 \mathrm{mg} \mathrm{L}^{-1}$, para solos de $\mathrm{CT}_{\mathrm{f}}$ e $\mathrm{CT}_{\mathrm{fa}}$, respectivamente.
\end{abstract}

Palavras-chave: concentração iônica, TDR, manejo da fertirrigação

\section{Relationship between potassium in the soil solution, soil water content and bulk electrical conductivity}

\begin{abstract}
The objective of this work was to evaluate the feasibility of estimating the potassium $(\mathrm{K})$ concentration in the soil solution, under laboratory conditions, for loamy $\left(\mathrm{CT}_{\mathrm{f}}\right)$ and sandy loam $\left(\mathrm{CT}_{\mathrm{fa}}\right)$ soils, through models that relate soil water content $(\theta)$, bulk electrical conductivity (CEa) and soil solution electrical conductivity (CEw). Five potassium chloride solutions with electrical conductivities of 1.0, 2.5, 4.0, 5.5 and $7.0 \mathrm{dS} \mathrm{m}^{-1}$, were applied in a soil packed in PVC columns in order to obtain five soil water contents $(17.0 \pm 1.4,19.0 \pm 1.8,21.4 \pm 2.2,23.4 \pm 2.2$ and $26.0 \pm 3.0 \%$, on volume basis). Readings of $\theta$ and CEa were obtained by using time domain reflectometry (TDR) equipment and soil solution was extracted by a water sampler to determine CEw and K. Three models were fitted to the data by an electronic sheet. It is possible to estimate potassium concentration in the soil solution from $\theta$ and CEa under laboratory conditions by the models of Rhoades et al. (1976), Vogeler et al. (1996) and Mualen \& Friedman (1991) using a potential relationship between CEw and K in the range of 0 to 60 and 0 to $120 \mathrm{mg} \mathrm{L}^{-1}$, for soils of $\mathrm{CT}_{\mathrm{f}}$ and $\mathrm{CT}_{\mathrm{fa}}$, respectively.
\end{abstract}

Key words: ionic concentration, TDR, fertirrigation management

1 Extraído da Tese de Doutorado do primeiro autor

2 Escola Agrotécnica Federal de Salinas, Fazenda Varginha, km 02, Rodovia Salinas-Taiobeiras, CP 71, CEP 39560-000, Salinas, MG. Fone: (38) 3841 - 1599 E-mail: gessionei@gmail.com

${ }^{3}$ Embrapa Mandioca e Fruticultura, CP 07, CEP 44380-000, Cruz das Almas, BA. Fone: (75) 3621 - 8021. E-mail: ecoelho@cnpmf.embrapa.br; tibérioss@hotmail.com

${ }^{4}$ DEA/UFV, CEP 36570-000, Viçosa, MG. Fone: (31) 3899 - 1914. E-mail: mmramos@ufv.br 


\section{INTRODUÇÃO}

A técnica de fertirrigação vem sendo utilizada, em todo o País, especialmente na Região Nordeste, em pólos agrícolas de produção de frutas e hortaliças. O uso desta técnica é crescente, sobretudo em condições de irrigação localizada. Com a expansão do uso desta tecnologia, a demanda por informações voltadas para o seu correto manejo tem aumentado sensivelmente; não obstante, tais informações ainda são escassas (Folegatti, 1999).

Os estudos a respeito de fertirrigação têm-se direcionado para a definição de freqüências de aplicação de fertilizantes e de doses e fontes de nutrientes, para condição de fertirrigação; aliado a isto, a preocupação com possíveis impactos do uso intensivo dessa técnica sobre o solo e a água, face à possibilidade de contaminação de lençóis freáticos devido à lixiviação de sais e de ocorrência de desbalanço químico e de alterações no pH do solo, é cada vez maior (Pinto, 2001).

O monitoramento de íons no solo constitui-se em um ponto chave no manejo da fertirrigação. Dessa forma, a implementação de um sistema automatizado capaz de monitorar, de forma contínua, a distribuição de água e de íons em áreas agrícolas, faz-se necessária para o correto manejo da fertirrigação nos solos cultivados. Uma alternativa viável para tal fim, é a técnica da Reflectometria no Domínio do Tempo (TDR) que, além de possibilitar, com o uso de uma única sonda, a determinação simultânea da umidade $(\theta)$ e da condutividade elétrica aparente do solo (CEa), em tempo real e de forma automatizada, rápida e com o mínimo distúrbio da estrutura do solo viabiliza, de forma indireta, o conhecimento da condutividade elétrica da solução do solo (CEw) e da concentração de nutrientes nesta solução (Dalton et al., 1984; Wraith \& Das, 1998; Mmolawa \& Or, 2000; Muñoz-Carpena et al. 2001; Noborio, 2001).

A técnica da TDR foi aplicada, inicialmente, ao solo, por Topp \& Davis (1980) objetivando medir a sua umidade, com base no tempo de deslocamento de pulsos eletromagnéticos, através de uma linha de transmissão (sonda), composta de hastes de aço de comprimento conhecido, introduzidas no solo (Tommaselli \& Bacchi, 2001). Posteriormente, esta técnica foi utilizada com sucesso em várias pesquisas, objetivando-se a determinação da $\theta$ e CEa (Rhoades et al., 1976; Rhoades et al., 1989; Vogeler et al. (1996); Heimovaara et al., 1995; Persson \& Uvo, 2003), a modelagem da dinâmica de água e solutos iônicos na zona radicular (Mmolawa \& Or, 2000), a identificação do perfil de extração de água pelo sistema radicular das culturas (Coelho \& Or, 1996) e a estimativa da evapotranspiração das culturas (Ward \& Dunin, 2001).

Wraith \& Das (1998) e Persson \& Uvo (2003) relatam que, devido à relação existente entre a $\mathrm{CEa}$ e a $\mathrm{CEw}$ e entre $\mathrm{CEw}$ e concentração iônica (Ci), é possível usar a TDR para monitorar a distribuição de água e solutos iônicos no solo; contudo, a TDR não determina, de forma direta, CEw e Ci, mas diversos modelos propostos na literatura relacionam $\theta, \mathrm{CEa}$ e CEw (Rhoades et al., 1976; Rhoades et al., 1989; Mualen \& Friedman, 1991; Heimovaara et al., 1995; Vogeler et al., 1996). Pesquisas recentes revelaram que é possível relacionar, satisfatoriamente, a CEw com a Ci (Wraith \& Das, 1998; Mmolawa \& Or, 2000; Muñoz-Carpena et al. 2001). Heimovaara et al. (1995) e Vogeler et al. (1996) relatam que a maioria dos autores concorda que é possível relacionar a CEw estimada com a Ci de um íon predominante na solução do solo, obtendo-se bons ajustes para modelos lineares; mas, estudos revelaram a necessidade de calibração de modelos para tal fim (Wraith \& Das, 1998; Persson \& Uvo, 2003).

Desenvolveu-se este trabalho objetivando-se avaliar, para condições de laboratório, a viabilidade de se estimar a concentração de potássio na solução do solo $(\mathrm{K})$, para solos aluviais de textura franca $\left(\mathrm{CT}_{\mathrm{f}}\right)$ e franco-arenosa $\left(\mathrm{CT}_{\mathrm{fa}}\right)$, a partir de dados de $\theta$ e CEa, determinados por meio da técnica da TDR.

\section{MATERIAL E MÉTODOS}

O experimento foi desenvolvido com um solo aluvial, coletado no Município de Nova Porteirinha, MG, situado nas seguintes coordenadas geográficas: altitude $533 \mathrm{~m}$, latitude $15^{\circ} 48^{\prime} 09^{\prime \prime}$ e longitude $43^{\circ} 18^{\prime} 32^{\prime \prime}$. A coleta do solo foi efetuada por camada de $0,20 \mathrm{~m}$ de espessura, a partir da superfície do solo até $0,80 \mathrm{~m}$ de profundidade, o que resultou em duas classes texturais para serem estudadas (Tabela 1). O solo coletado na camada superficial $(0-0,20 \mathrm{~m})$, resultou na classe textural franco-arenosa $\left(\mathrm{CT}_{\mathrm{fa}}\right)$, ao passo que o solo coletado na profundidade de $0,20-0,80 \mathrm{~m}$ resultou na classe textural franca $\left(\mathrm{CT}_{\mathrm{f}}\right)$. O solo foi secado ao ar, destorroado e peneirado em peneira com malha de $2 \mathrm{~mm}$. Segmentos de tubo de PVC com $0,19 \mathrm{~m}$ de altura e $0,10 \mathrm{~m}$ de diâmetro foram preenchidos com $1,90 \mathrm{~kg}$ de terra fina secada ao ar (TFSA), até a altura de $0,17 \mathrm{~m}$, perfazendo um volume de solo no tubo de PVC de $0,00133518 \mathrm{~m}^{3}$.

O experimento consistiu de cinco soluções de cloreto de potássio, referentes a cinco condutividades elétricas (CE) $(1,0$; 2,5, 4,0, 5,5 e 7,0 dS m $\mathrm{m}^{-1}$ ), que foram aplicadas sobre o solo, de forma a se obter cinco umidades do solo $(\theta)(17,0 \pm 1,4$, $19,0 \pm 1,8,21,4 \pm 2,2,23,4 \pm 2,2$ e $26,0 \pm 3,0 \%$, em base de volume), resultando em 25 tratamentos (Figura 1), que foram repetidos duas vezes, para a $\mathrm{CT}_{\mathrm{f}}$, obtendo-se 50 observações enquanto para a $\mathrm{CT}_{\text {fa }}$, os tratamentos não foram repetidos e, portanto, obtiveram-se apenas 25 observações.

Tabela 1. Características físico-hídricas das classes texturais do solo estudado

\begin{tabular}{|c|c|c|c|c|c|c|c|}
\hline \multirow{3}{*}{ Classe Textural } & \multirow[t]{2}{*}{ Areia } & \multirow[t]{2}{*}{ Silte } & \multirow[t]{3}{*}{ Argila } & \multicolumn{2}{|c|}{ Densidade } & \multicolumn{2}{|c|}{ Umidade } \\
\hline & & & & Solo & Partículas & $-10 \mathrm{kPa}$ & $-1.500 \mathrm{kPa}$ \\
\hline & \multicolumn{2}{|r|}{$\left(\mathrm{g} \mathrm{kg}^{-1}\right)$} & & \multicolumn{2}{|c|}{$\left(\mathrm{kg} \mathrm{dm}^{-3}\right)$} & \multicolumn{2}{|c|}{$\left(\mathrm{kg} \mathrm{kg}^{-1}\right)$} \\
\hline Franca & 427,3 & 417,3 & 155,3 & 1,47 & 2,39 & 0,2226 & 0,1608 \\
\hline Franco-arenosa & 544,0 & 355,0 & 101,0 & 1,45 & 2,43 & 0,2013 & 0,1503 \\
\hline
\end{tabular}


A umidade do solo referente à umidade residual $\left(\theta_{\mathrm{r}}\right)$, foi obtida pelo método padrão de estufa (Bernardo, 1995), possibilitando a correta complementação do nível de água do solo para os níveis desejados.

A fim de se conhecer a relação entre concentrações de cloreto de potássio $(\mathrm{KCl})$ e condutividades elétricas $(\mathrm{CE})$ de soluções de $\mathrm{KCl}$, realizou-se um ensaio preliminar que consistiu na preparação de soluções de $\mathrm{KCl}$, mediante a solubilização de quantidades crescentes de $\mathrm{KCl}$ em água destilada; em seguida, determinaram-se as CE por meio de condutivímetro de mesa. Submetendo-se os valores de concentrações de $\mathrm{KCl}$ e $\mathrm{CE}$ das soluções de $\mathrm{KCl}$ à análise de regressão, chegou-se à Eq. 1:

$$
\begin{gathered}
{[\mathrm{KCl}]=\frac{\mathrm{CE}-0,0900}{1,6257}} \\
\mathrm{com} \mathrm{R}^{2}=1,00
\end{gathered}
$$

em que:

$\mathrm{KCl}$ e CE correspondem, respectivamente, a concentrações de cloreto de potássio nas soluções de cloreto de potássio,

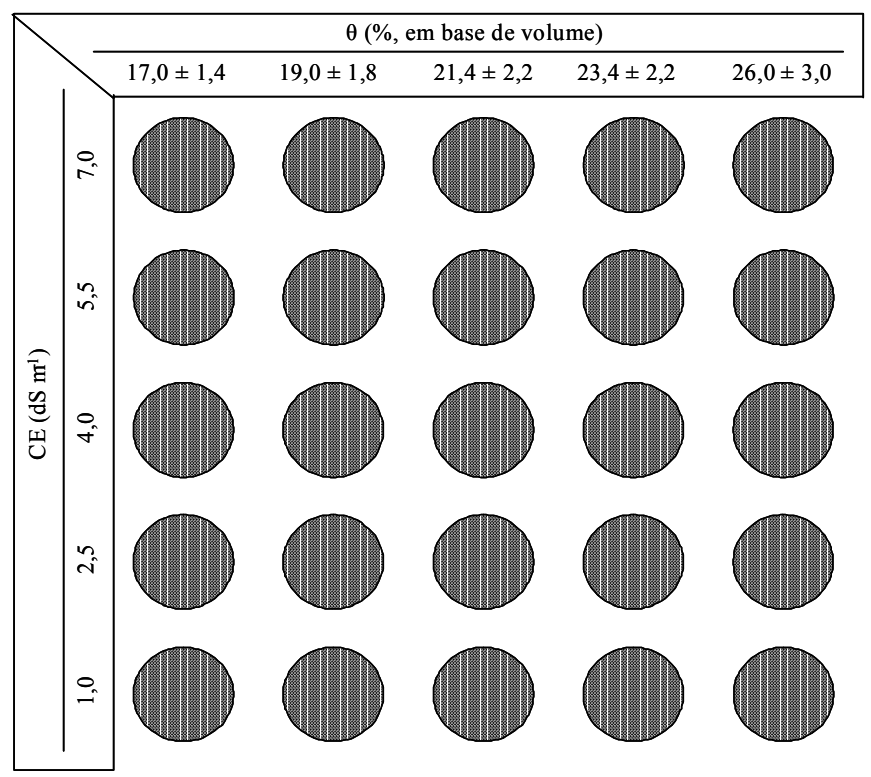

Figura 1. Croqui dos tratamentos provenientes da combinação de cinco $\theta$ com cinco $\mathrm{CE}$

em $\mathrm{g} \mathrm{L}^{-1}$, e condutividades elétricas das soluções de cloreto de potássio, em $\mathrm{dS} \mathrm{m}^{-1}$.

A umidade do solo foi determinada por meio da equação de Ledieu et al. (1986) e a CEa por meio da equação proposta por Giese \& Tiemann (1975), cujos valores foram corrigidos para a temperatura de $25^{\circ} \mathrm{C}$, conforme a Eq. 2 (Richards, 1954):

$$
\mathrm{f}_{\mathrm{T}}=1+\frac{(25-\mathrm{T})}{49,7}+\frac{(25-\mathrm{T})^{2}}{3.728}
$$

\footnotetext{
${ }^{1}$ A menção da marca do equipamento não representa endosso pelos autores
}

$$
\text { para } 20<\mathrm{T}<47^{\circ} \mathrm{C}
$$

em que:

$\mathrm{f}_{\mathrm{T}}$ - fator de correção da CE quanto aos efeitos da temperatura, adimensional

$\mathrm{T}$ - corresponde à temperatura da solução do solo, em ${ }^{\circ} \mathrm{C}$.

Logo após as leituras de $\theta$ e CEa pela TDR, retirou-se a sonda do solo e se introduziu um segmento de tubo PVC de $12 \mathrm{~mm}$ até a profundidade correspondente ao comprimento da haste da sonda. No orifício feito pelo tubo de PVC, introduziu-se um extrator de solução do solo, provido de cápsula de cerâmica, devidamente limpo, e se lhe aplicou uma sucção equivalente a $-70 \mathrm{kPa}$ (Rhoades et al., 1976; Heimovaara et al., 1995; Wraith \& Das, 1998; Vogeler et al., 1996; MuñozCarpena et al. 2001); após a sucção de cerca de $15 \mathrm{~mL}$ de solução do solo, retirou-se o extrator, homogeneizou-se a solução armazenando-a em frascos de vidro previamente lavados para posterior determinação da sua condutividade elétrica $(\mathrm{CEw})$, por meio de condutivímetro de mesa.

Preliminarmente, seis modelos matemáticos foram avaliados quanto à sua capacidade de relacionar $\theta, \mathrm{CEa}$ e CEw; a partir desta avaliação, selecionaram-se os três modelos que melhor se ajustaram aos dados observados de $\theta$, CEa e CEw, para fins de estimativa da concentração de $\mathrm{K}$ na solução do solo (Tabela 2).

A umidade de saturação $\left(\theta_{\mathrm{s}}\right)$ foi obtida conforme a Eq. 3:

$$
\theta \mathrm{s}=1-\left(\frac{\mathrm{Ds}}{\mathrm{Dp}}\right)
$$

em que:

Ds e Dp - correspondem, respectivamente, à densidade do solo e de partículas e são expressas em $\mathrm{kg} \mathrm{dm}^{-3}$

Os ajustes dos modelos utilizados para se estimar a concentração de K na solução do solo (Tabela 2), foram otimizados explicitando-se a variável CEw (Tabela 3); em segui$\mathrm{da}$, relacionaram-se os valores medidos de $\mathrm{CEw}$ e de $\mathrm{K}$, obtendo-se equações do tipo potência (Mmolawa \& Or, 2000) e linear (Muñoz-Carpena et al., 2001; Heimovaara et al., 1995; Vogeler et al, 1996; Vogeler et al., 1997 e Wraith \& Das, 1998):

$$
\mathrm{CEw}=\alpha \mathrm{K}^{\rho}
$$

$$
\mathrm{CEw}=\alpha+\rho \mathrm{K}
$$

em que:

$$
\alpha \text { e } \rho \text { - parâmetros adimensionais das equações }
$$

Substituindo-se as Eqs. 4 e 5 nas Eqs. 9, 10 e 11, obtive- 
Tabela 2. Modelos utilizados para se estimar a concentração de $\mathrm{K}$ na solução do solo ${ }^{1}$, na sua forma original

\begin{tabular}{|c|c|c|c|}
\hline Autor & Modelo & Parâmetros ${ }^{2}$ & Equação $n^{0}$ \\
\hline Rhoades et al. (1976) & $\mathrm{CEa}=\left(\mathrm{a} \theta^{2}+\mathrm{b} \theta\right) \mathrm{CEw}+\mathrm{CEs}$ & $\mathrm{a}, \mathrm{b}, \mathrm{CEs}^{3}$ & (6) \\
\hline Mualen \& Friedman (1991) & $\mathrm{CEa}=\mathrm{CEw}\left(\frac{\theta^{\beta}}{\theta \mathrm{s}}\right)+\mathrm{CEs}$ & $\beta, \mathrm{CES}^{3}$ & (7) \\
\hline Vogeler et al. (1996) & $\mathrm{CEa}=(\mathrm{c} \theta-\mathrm{d}) \mathrm{CEw}+(\mathrm{a} \theta-\mathrm{b})$ & $a, b, c, d$ & (8) \\
\hline \multirow{2}{*}{\multicolumn{4}{|c|}{ 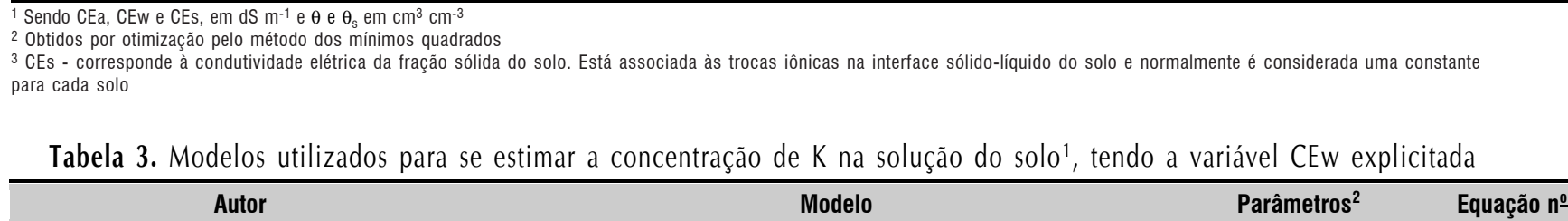 }} \\
\hline & & & \\
\hline Rhoades et al. (1976) & $\mathrm{CEw}=\frac{(\mathrm{CEa}-\mathrm{CEs})}{\left(\mathrm{a} \theta^{2}+\mathrm{b} \theta\right)}$ & $\mathrm{a}, \mathrm{b}, \mathrm{CEs}^{3}$ & (9) \\
\hline Mualen \& Friedman (1991) & $\mathrm{CEw}=\frac{(\mathrm{CEa}-\mathrm{CEs}) \theta \mathrm{s}}{\theta^{\beta}}$ & $\beta, \mathrm{CES}^{3}$ & (10) \\
\hline Vogeler et al. (1996) & $\mathrm{CEw}=\frac{[\mathrm{CEa}-(\mathrm{a} \theta-\mathrm{b})]}{(\mathrm{c} \theta-\mathrm{d})}$ & $a, b, c, d$ & (11) \\
\hline
\end{tabular}

1 Sendo CEa, CEW e CEs, em dS m $\mathrm{m}^{-1} \mathrm{e} \theta$ e $\theta_{\mathrm{s}} \mathrm{em} \mathrm{cm}^{3} \mathrm{~cm}^{-3}$

2 Obtidos por otimização pelo método dos mínimos quadrados

3 CEs - corresponde à condutividade elétrica da fração sólida do solo. Está associada às trocas iônicas na interface sólido-líquido do solo e normalmente é considerada uma constante para cada solo

Tabela 4. Modelos adaptados para se estimar a concentração de $\mathrm{K}$ na solução do solo ${ }^{1}$, em mg $\mathrm{L}^{-1}$, tendo a variável $\mathrm{K}$ explicitada

\begin{tabular}{|c|c|c|c|c|}
\hline Autor & Modelo & & Parâmetros ${ }^{2}$ & Equaçãa $n^{0}$ \\
\hline $\begin{array}{l}\text { Rhoades et al. (1976) adaptado para a relação } \\
\text { CEw-K do tipo potência }\end{array}$ & $\mathrm{K}=\left[\frac{1}{\alpha} \frac{(\mathrm{CEa}-\mathrm{CEs})}{\left(\mathrm{a} \theta^{2}+\mathrm{b} \theta\right)}\right]^{\frac{1}{\rho}}$ & & $a, b, \mathrm{CEs}^{3}, \alpha, \rho$ & (12) \\
\hline $\begin{array}{l}\text { Rhoades et al. (1976) adaptado para a relação } \\
\text { CEw-K do tipo linear }\end{array}$ & $\mathrm{K}=\left[\frac{(\mathrm{CEa}-\mathrm{CEs})}{\left(\mathrm{a} \theta^{2}+\mathrm{b} \theta\right)}-\alpha\right] \frac{1}{\rho}$ & $\frac{1}{\rho}$ & $a, b, C E s^{3}, \alpha, \rho$ & (13) \\
\hline $\begin{array}{l}\text { Mualen \& Friedman (1991) adaptado para a } \\
\text { relação CEw-K do tipo potência }\end{array}$ & $\mathrm{K}=\left[\frac{1}{\alpha} \frac{(\mathrm{CEa}-\mathrm{CEs}) \theta \mathrm{s}}{\theta^{\beta}}\right]$ & ]$^{\frac{1}{\rho}}$ & $\beta, \mathrm{CEs}^{3}, \alpha, \rho$ & (14) \\
\hline $\begin{array}{l}\text { Mualen \& Friedman (1991) adaptado para a } \\
\text { relação CEw-K do tipo linear }\end{array}$ & $\mathrm{K}=\left[\frac{(\mathrm{CEa}-\mathrm{CEs}) \theta \mathrm{s}}{\theta^{\beta}}-\alpha\right]$ & ]$\frac{1}{\rho}$ & $\beta, \mathrm{CEs}^{3}, \alpha, \rho$ & (15) \\
\hline $\begin{array}{l}\text { Vogeler et al. (1996) adaptado para a relação } \\
\text { CEw-K do tipo potência }\end{array}$ & $\mathrm{K}=\left\{\frac{1}{\alpha} \frac{[\mathrm{CEa}-(\mathrm{a} \theta-\mathrm{b})]}{(\mathrm{c} \theta-\mathrm{d})}\right\}$ & \}$^{\frac{1}{\rho}}$ & $a, b, c, d, \alpha, \rho$ & (16) \\
\hline $\begin{array}{l}\text { Vogeler et al. (1996) adaptado para a relação } \\
\text { CEw-K do tipo linear }\end{array}$ & $\mathrm{K}=\left\{\frac{[\mathrm{CEa}-(\mathrm{a} \theta-\mathrm{b})]}{(\mathrm{c} \theta-\mathrm{d})}-\alpha\right\}$ & \}$\frac{1}{\rho}$ & $a, b, c, d, \alpha, \rho$ & (17) \\
\hline
\end{tabular}

${ }_{1}^{1}$ Sendo CEa, CEw e CEs, em dS $\mathrm{m}^{-1}$ e $\theta$ e $\theta_{\mathrm{s}}$ em $\mathrm{cm}^{3} \mathrm{~cm}^{-3}$

2 Obtidos por otimização pelo método dos mínimos quadrados

${ }^{3}$ CEs corresponde à condutividade elétrica da fração sólida do solo. Está associada às trocas iônicas na interface sólido-líquido do solo e normalmente é considerada uma constante para cada solo

ram-se as Eqs. 12, 13, 14, 15, 16 e 17 (Tabela 4) que, em seguida, tiveram seus ajustes otimizados, explicitando K.

A avaliação dos modelos quanto à sua capacidade de relacionar $\theta, \mathrm{CEa}$ e $\mathrm{K}$, com vistas à estimativa de $\mathrm{K}$ a partir de dados de $\theta$ e CEa obtidos por meio da técnica da TDR, foi feita com base no coeficiente de concordância (D), proposto por Willmontt (1981) (Eq. 18), no coeficiente de determinação $\left(\mathrm{R}^{2}\right)$ e no grau de ajuste da variável dependente $(\mathrm{Y})$ com a variável independente $(\mathrm{X})$, mediante o estabelecimento de uma equação do tipo $\mathrm{Y}=\mathrm{aX}$, após otimização de seus ajustes aos dados medidos, por meio de planilha eletrônica.

$$
D=1-\left[\frac{\sum_{i=1}^{n}\left(M_{i}-E_{i}\right)^{2}}{\sum_{i=1}^{n}\left(\left|M_{i}-\bar{M}\right|+\left|E_{i}-\bar{M}\right|\right)^{2}}\right]
$$

em que:

$\mathrm{M}_{\mathrm{i}}$ - valor medido de ordem $\mathrm{i}$

$E_{i}$ - valor estimado de ordem $i$

$\overline{\mathrm{M}}$ - média dos valores medidos

$\mathrm{n}$ - número de valores medidos

O coeficiente de concordância (D) expressa a exatidão dos 
valores estimados em relação aos valores medidos, variando de zero, que indica nulidade, a um que indica perfeita exatidão.

\section{RESULTADOS E DISCUSSÃO}

Os valores dos parâmetros dos três modelos que melhor se ajustaram aos dados observados e seus respectivos coeficientes de determinação $\left(\mathrm{R}^{2}\right)$, assim como, os valores do coeficiente de concordância (D), para os solos de classes texturais franca $\left(\mathrm{CT}_{\mathrm{f}}\right)$ e franco-arenosa $\left(\mathrm{CT}_{\mathrm{fa}}\right)$, são apresentados na Tabela 5. De modo geral, nota-se que os modelos estimaram melhor a CEw a partir dos dados do solo de $\mathrm{CT}_{\mathrm{f}}$ $\left(\mathrm{R}^{2}=0,80\right.$ e $\left.\mathrm{D}=0,94\right)$ que a partir dos dados do solo de $\mathrm{CT}_{\mathrm{fa}}$ $\left(\mathrm{R}^{2}=0,77\right.$ e $\left.\mathrm{D}=0,93\right)$. Percebe-se, ainda, equivalência entre os três modelos para o solo de $\mathrm{CT}_{\mathrm{f}}\left(\mathrm{R}^{2}=0,80\right.$ e $\left.\mathrm{D}=0,94\right)$, ao passo que, para o solo de $\mathrm{CT}_{\text {fa }}$, o modelo de Vogeler et al. (1996) se sobressaiu frente aos demais, com $\mathrm{R}^{2}=0,80$, contra 0,77 e 0,75 e $\mathrm{D}=0,94$, contra 0,93 e 0,92 , dos modelos de Rhoades et al. (1976) e de Mualen \& Friedman (1991) respectivamente.

Confeccionada com os valores de CEw estimados a partir dos valores dos parâmetros contidos na Tabela 5, a Figura 2, referente ao solo de classe textural franca $\left(\mathrm{CT}_{\mathrm{f}}\right)(\mathrm{A})$ e ao solo de classe textural franco-arenosa $\left(\mathrm{CT}_{\mathrm{fa}}\right)(\mathrm{B})$, esboça a

A.

B.
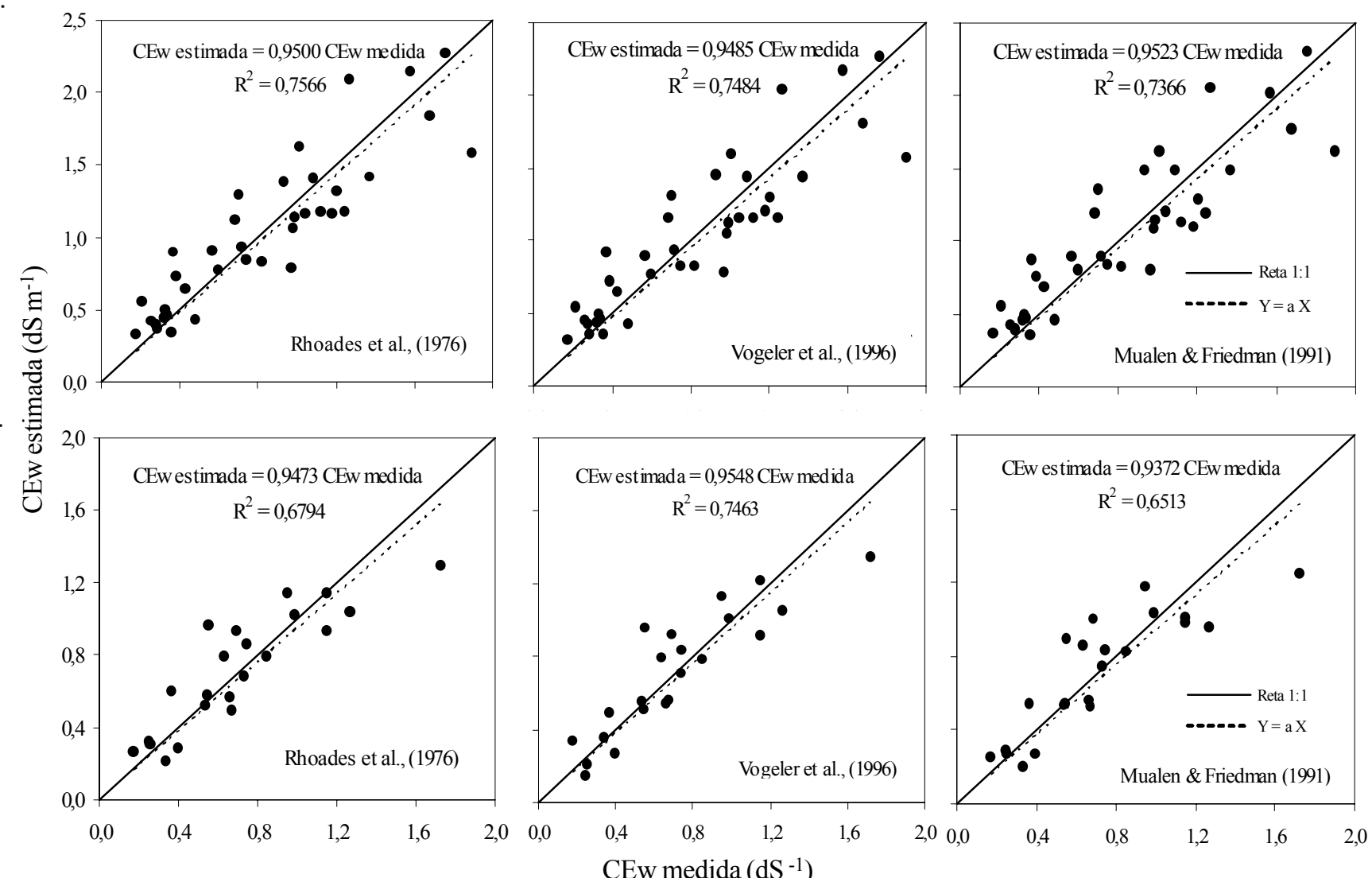

Figura 2. Relação entre a CEw medida e estimada pelos modelos que a relacionam com CEa e $\theta$, para os solos de classe textural franca $\left(C T_{f}\right)(A)$ e de classe textural franco-arenosa $\left(\mathrm{CT}_{\mathrm{fa}}\right)(\mathrm{B})$

Tabela 5. Coeficiente de concordância (D), parâmetros e coeficientes de determinação $\left(R^{2}\right)$ dos três modelos que melhor se ajustaram aos dados observados de $\theta$, CEa e CEw, para os solos de $\mathrm{CT}_{\mathrm{f}}$ e $\mathrm{CT}_{\mathrm{f} \mathrm{f}^{\prime}}$ na estimativa da CEw

\begin{tabular}{|c|c|c|c|c|c|c|c|c|}
\hline \multirow{2}{*}{ Autor } & \multirow{2}{*}{ D } & \multicolumn{6}{|c|}{ Parâmetros } & \multirow{2}{*}{$\mathbf{R}^{2}$} \\
\hline & & a & b & c & d & CEs & $\beta$ & \\
\hline \multicolumn{9}{|c|}{ Solo de classe textural franca $\left(\mathrm{CT}_{\mathrm{f}}\right)$} \\
\hline Rhoades et al. (1976) & 0,9404 & 4,4152 & 0,2958 & - & - & 0,0902 & - & 0,8018 \\
\hline Vogeler et al. (1996) & 0,9384 & 0,1930 & $-0,0488$ & 2,0014 & 0,1577 & - & - & 0,7960 \\
\hline Mualen \& Friedman (1991) & 0,9371 & - & - & - & - & 0,0844 & 1,4955 & 0,7943 \\
\hline \multicolumn{9}{|c|}{ Solo de classe textural franco-arenosa $\left(\mathrm{CT}_{\mathrm{fa}}\right)$} \\
\hline Rhoades et al. (1976) & 0,9252 & $-2,5186$ & 2,4133 & - & - & 0,0797 & - & 0,7667 \\
\hline Vogeler et al. (1996) & 0,9389 & 2,2220 & 0,3552 & $-1,0447$ & $-0,5858$ & - & - & 0,7996 \\
\hline Mualen \& Friedman (1991) & 0,9174 & - & - & - & - & 0,0911 & 1,2496 & 0,7463 \\
\hline
\end{tabular}


A.
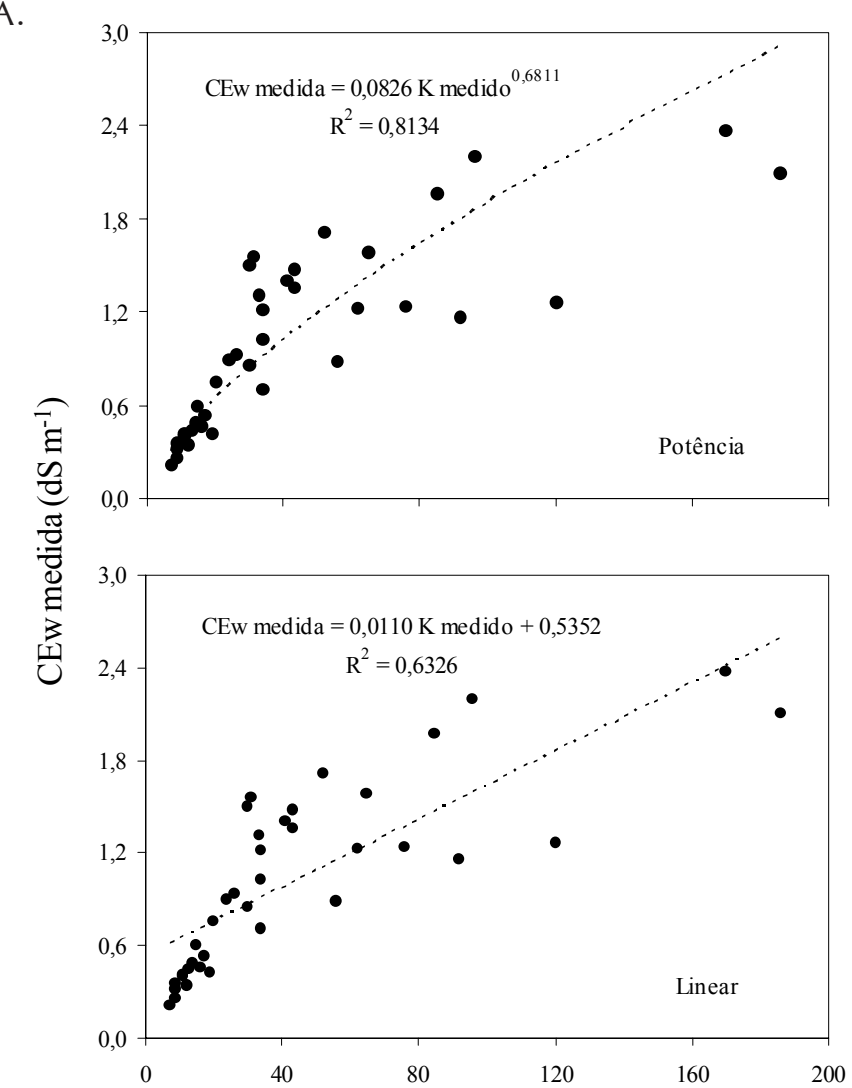

B.
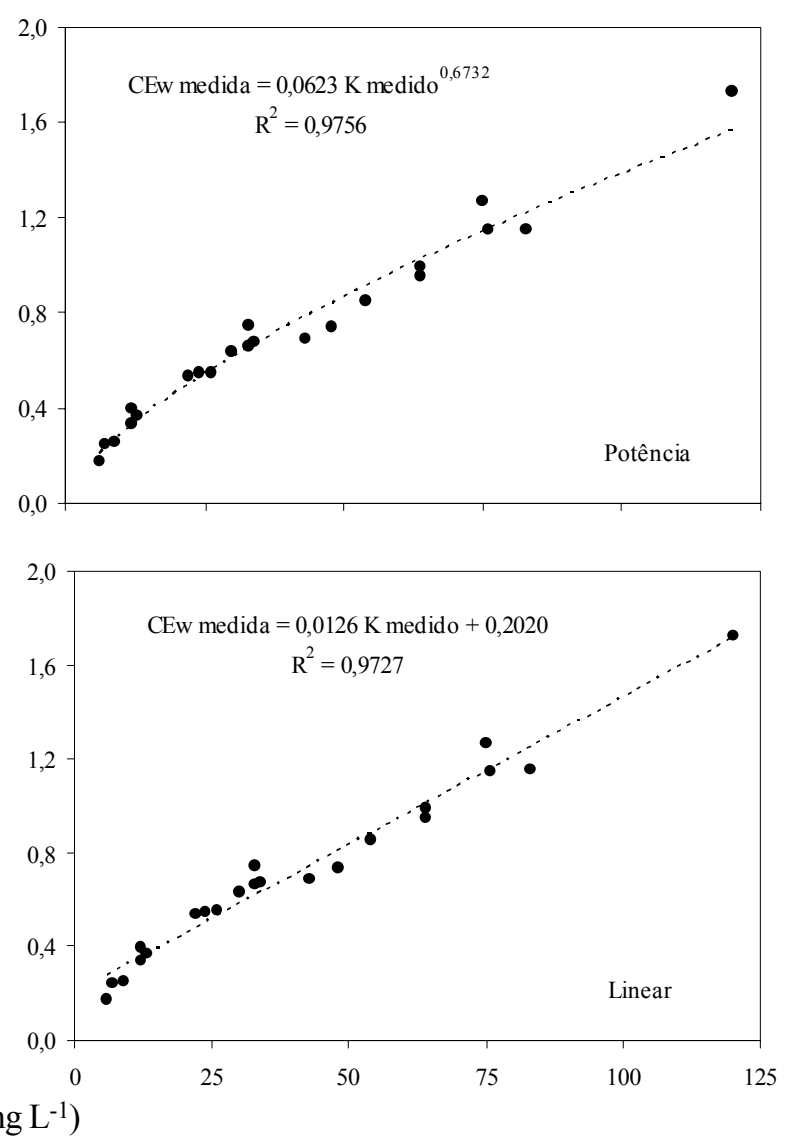

Figura 3. Relação entre CEw e K para os solos de classe textural franca $\left(C_{\mathrm{f}}\right)(\mathrm{A})$ e de classe textural franco-arenosa $\left(C \mathrm{C}_{\mathrm{fa}}\right)(\mathrm{B})$

mesma tendência observada nos resultados apresentados na Tabela 5.

Relações do tipo potência e linear entre as variáveis CEw e K medidas na solução do solo, são mostradas na Figura 3, referente ao solo de classe textural franca $\left(\mathrm{CT}_{\mathrm{f}}\right)(\mathrm{A})$ e ao solo de classe textural franco-arenosa $\left(\mathrm{CT}_{\mathrm{fa}}\right)(\mathrm{B})$. Para o solo de $\mathrm{CT}_{\mathrm{f}}$, o modelo que melhor explicou essa relação foi o do tipo potência, com $\mathrm{R}^{2}=0,81$; já para o solo de $\mathrm{CT}_{\mathrm{fa}}$, os modelos linear e potência foram equivalentes na explicação desta relação, apesar do modelo tipo potência ser ligeiramente superior $\left(\mathrm{R}^{2}=0,98\right)$ ao modelo tipo linear $\left(\mathrm{R}^{2}=0,97\right)$. O ajuste entre $\mathrm{CEw}$ e $\mathrm{K}$, para o solo de $\mathrm{CT}_{\mathrm{f}}$, está de acordo com Mmolawa \& Or (2000) mas difere daqueles obtidos por Heimovaara et al. (1995), Vogeler et al. (1996), Vogeler et al. (1997) e Wraith \& Das (1998), Muñoz-Carpena et al. (2001), que foram do tipo linear; porém para o solo de $\mathrm{CT}_{\mathrm{fa}} \mathrm{o}$ ajuste entre $\mathrm{CEw}$ e $\mathrm{K}$ está de acordo com todos esses autores, visto que tanto o modelo potência quanto o linear, explicaram bem esta relação. Vogeler et al. (1997) relatam que, para baixas concentrações iônicas, a CEw se relaciona, de forma linear, com a concentração de determinado sal presente na solução do solo.

Vê-se, na Tabela 6, os valores dos parâmetros e dos coeficientes de determinação $\left(\mathrm{R}^{2}\right)$ dos modelos adaptados e avaliados para os solos de $\mathrm{CT}_{\mathrm{f}}$ e $\mathrm{CT}_{\mathrm{fa}}$. Evidencia-se notável supremacia do ajuste dos dados do solo de textura franco-arenosa $\left(\mathrm{CT}_{\mathrm{fa}}\right)$ e com menor percentual de partículas finas e, portanto, com menor superfície específica, por todos os modelos $\left(\mathrm{R}^{2}=0,79\right.$ e $\left.\mathrm{D}=0,94\right)$, comparativamente àquele obtido para o solo de textura franca $\left(\mathrm{CT}_{\mathrm{f}}\right)$ e com maior percentual de partículas finas $\left(\mathrm{R}^{2}=0,60\right.$ e $\left.\mathrm{D}=0,85\right)$. Tais constatações podem ser devidas às características físicas e/ou físico-químicas do solo, tal como a capacidade de troca de cátions (CTC), que pode atuar com maior ou menor intensidade na adsorção e/ou dessorção de cátions.

Observa-se, para o solo de $\mathrm{CT}_{\text {fa }}$, que uma estimativa melhor da concentração de $\mathrm{K}$ na solução do solo a partir de dados de umidade $(\theta)$ e condutividade elétrica aparente $(\mathrm{CEa})$, é obtida quando se introduz, nos modelos adaptados, uma relação do tipo potência entre $\mathrm{CEw}$ e $\mathrm{K}\left(\mathrm{R}^{2}=0,81\right.$ e $\left.\mathrm{D}=0,94\right)$; mas, para o solo de $\mathrm{CT}_{\mathrm{f}}$, percebe-se que as estimativas de $\mathrm{K}$ foram equivalentes quando se introduziu, nos modelos adaptados, qualquer uma das relações entre CEw e K (tipo linear ou potência), exceto para o modelo de Rhoades et al. (1976), adaptado com uma relação entre CEw e $\mathrm{K}$ do tipo potência, que foi superior aos demais, em cerca de $7 \%$, quanto ao $\mathrm{R}^{2}$, porém, quanto ao coeficiente de concordância (D), com valor médio igual a 0,85 , não se observa diferença considerável.

Em relação aos modelos adaptados, verifica-se que os de Rhoades et al. (1976) e de Vogeler et al. (1996) proporcionaram as melhores estimativas de concentração de $\mathrm{K}$ na solução do solo, a partir de dados de $\theta$ e CEa, para o solo de $\mathrm{CT}_{\mathrm{f}}$ e $\mathrm{CT}_{\text {fa }}$, respectivamente, com valores de $\mathrm{R}^{2}$ iguais a 0,66 e 0,82 e de $\mathrm{D}$ iguais a 0,85 e 0,95 . 
Tabela 6. Coeficiente de concordância (D), parâmetros e coeficientes de determinação $\left(\mathrm{R}^{2}\right)$, para o solo de $\mathrm{CT}_{\mathrm{f}}$ e de $\mathrm{CT}_{\mathrm{fa}}$, na estimativa da concentração de $K$ na solução do solo

\begin{tabular}{|c|c|c|c|c|c|c|c|c|c|c|}
\hline \multirow{2}{*}{ Autor } & \multirow{2}{*}{ D } & \multicolumn{8}{|c|}{ Parâmetros } & \multirow{2}{*}{$\mathrm{R}^{2}$} \\
\hline & & a & b & c & d & CES & $\beta$ & $\alpha$ & $\rho$ & \\
\hline \multicolumn{11}{|c|}{ Solo de classe textural franca $\left(\mathrm{CT}_{\mathrm{f}}\right)$} \\
\hline $\begin{array}{l}\text { Rhoades et al. (1976) adaptado para } \\
\text { a relação CEw-K do tipo potência }\end{array}$ & 0,8518 & 1,8504 & 0,9550 & - & - & 0,0920 & - & 0,0461 & 0,8425 & 0,6558 \\
\hline $\begin{array}{l}\text { Rhoades et al. (1976) adaptado para } \\
\text { a relação CEw-K do tipo linear }\end{array}$ & 0,8462 & 0,7820 & 0,0360 & - & - & 0,1276 & - & 0,9597 & 0,0995 & 0,5863 \\
\hline $\begin{array}{l}\text { Vogeler et al. (1996) adaptado para } \\
\text { a relação CEw-K do tipo potência }\end{array}$ & 0,8434 & 0,5178 & $-0,0508$ & 0,9259 & 0,0690 & - & - & 0,0459 & 0,9383 & 0,5808 \\
\hline $\begin{array}{l}\text { Vogeler et al. (1996) adaptado para } \\
\text { a relação CEw-K do tipo linear }\end{array}$ & 0,8448 & $-16,7941$ & $-1,5471$ & 0,9462 & 0,0804 & - & - & 18,1821 & 0,0358 & 0,5787 \\
\hline $\begin{array}{l}\text { Mualen \& Friedman (1991) adaptado para a } \\
\text { relação CEw-K do tipo potência }\end{array}$ & 0,8462 & - & - & - & - & 0,1565 & 1,9905 & 0,0485 & 0,9299 & 0,5864 \\
\hline $\begin{array}{l}\text { Mualen \& Friedman (1991) adaptado para a } \\
\text { relação CEw-K do tipo linear }\end{array}$ & 0,8460 & - & - & - & - & 0,1392 & 1,9058 & 0,2041 & 0,0303 & 0,5859 \\
\hline \multicolumn{11}{|c|}{ Solo de classe textural franco-arenosa $\left(\mathbf{C T}_{\mathrm{fa}}\right)$} \\
\hline $\begin{array}{l}\text { Rhoades et al. (1976) adaptado para a relação } \\
\text { CEw-K do tipo potência }\end{array}$ & 0,9432 & $-1,2478$ & 2,8999 & - & - & 0,0500 & - & 0,1023 & 0,4856 & 0,8040 \\
\hline $\begin{array}{l}\text { Rhoades et al. (1976) adaptado para a relação } \\
\text { CEw-K do tipo linear }\end{array}$ & 0,9254 & $-2,0016$ & 2,8238 & - & - & 0,0300 & - & 0,2777 & 0,0095 & 0,7619 \\
\hline $\begin{array}{l}\text { Vogeler et al. (1996) adaptado para a relação } \\
\text { CEw-K do tipo potência }\end{array}$ & 0,9465 & 2,2670 & 0,3560 & $-1,0400$ & $-0,5858$ & - & - & 0,0710 & 0,6342 & 0,8178 \\
\hline $\begin{array}{l}\text { Vogeler et al. (1996) adaptado para a relação } \\
\text { CEw-K do tipo linear }\end{array}$ & 0,9341 & 1,9918 & 0,2095 & $-0,0548$ & $-0,0373$ & - & - & $-0,9437$ & 0,1802 & 0,7852 \\
\hline $\begin{array}{l}\text { Mualen \& Friedman (1991) adaptado para a } \\
\text { relação CEw-K do tipo potência }\end{array}$ & 0,9429 & - & - & - & - & 0,0500 & 0,9196 & 0,0878 & 0,4851 & 0,8033 \\
\hline $\begin{array}{l}\text { Mualen \& Friedman (1991) adaptado para a } \\
\text { relação CEw-K do tipo linear }\end{array}$ & 0,9335 & - & - & - & - & $-3,5209$ & 0,0849 & 1,5617 & 0,0019 & 0,7830 \\
\hline
\end{tabular}

Tendo em vista o baixo desempenho apresentado pelos modelos adaptados na estimativa do $\mathrm{K}$ da solução do solo para o solo de $\mathrm{CT}_{\mathrm{f}}$ (Tabela 6), plotou-se o $\mathrm{K}$ estimado como função do $\mathrm{K}$ medido, por meio de uma função do tipo $\mathrm{Y}=\mathrm{aX}$, apenas para o modelo de Rhoades et al. (1976), adaptado com uma relação entre $\mathrm{CEw}$ e $\mathrm{K}$ do tipo potência, que foi o que melhor estimou o K da solução do solo. Esta relação pode ser observada na Figura 4, na qual se percebe, também, considerável dispersão dos dados medidos e estimados a partir de uma concentração de $\mathrm{K}$ na solução do solo superior a $60 \mathrm{mg}$ $\mathrm{L}^{-1}$. A referida dispersão, além de reforçar os baixos ajustes apresentados na Tabela 6 , revela a fragilidade desses modelos na estimativa daquele $\mathrm{K}$, quando a concentração desse íon na solução do solo é superior a $60 \mathrm{mg} \mathrm{L}^{-1}$ para solos com características físico-químicas similares às do solo de $\mathrm{CT}_{\mathrm{f}}$.

No trabalho de Nadler (1997), também se constatou aumento da dispersão dos dados com o incremento do teor de partículas menores; desta forma, o baixo ajuste dos modelos adaptados aos dados do solo de $\mathrm{CT}_{\mathrm{f}}$ e, conseqüentemente, com maior teor de silte e argila, comparativamente ao do solo de $\mathrm{CT}_{\text {fa }}$, pode ser devido às características físico-químicas desse solo sugerindo, portanto, que tais características influenciam na estimativa da concentração de $\mathrm{K}$ na solução do solo, com esses modelos.

$\mathrm{Na}$ Figura 5, o K estimado para o solo de $\mathrm{CT}_{\text {fa }}$, por meio dos modelos adaptados com uma relação entre CEw e $\mathrm{K}$ do

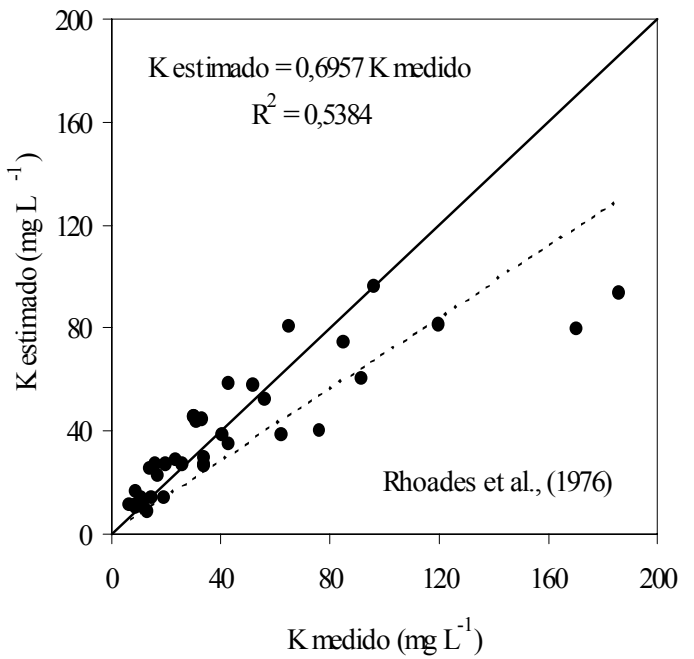

Figura 4. Relação entre o K medido e estimado pela equação 12, para o solo de $\mathrm{CT}_{\mathrm{f}}$

tipo potência (A) e linear (B), é plotado como função da concentração de K medida na solução do solo. Nota-se que os modelos adaptados com uma relação entre $\mathrm{CEw}$ e $\mathrm{K}$ do tipo potência (Figura $5 \mathrm{~A}$ ) proporcionaram melhor estimativa da concentração de $\mathrm{K}$ na solução do solo $\left(\mathrm{R}^{2}=0,78\right)$, que quando adaptados com uma relação do tipo linear $\left(\mathrm{R}^{2}=0,72\right)(\mathrm{Fi}-$ gura 5B). Para este solo, o modelo adaptado de Vogeler et al. 
A.
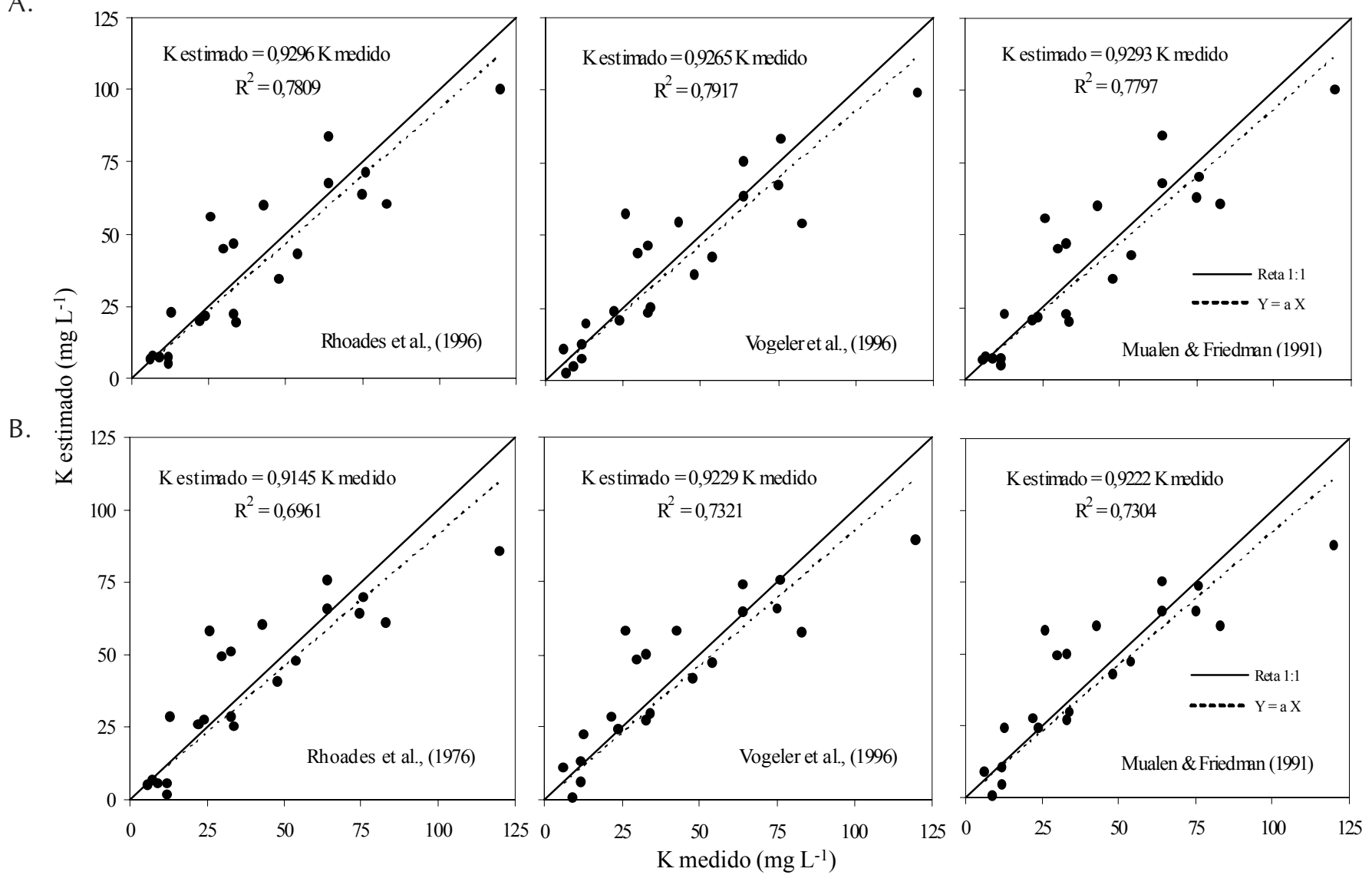

Figura 5. Relação entre o K medido e estimado, para o solo de $\mathrm{CT}_{\mathrm{fa}}$

Tabela 7. Coeficiente de concordância (D), parâmetros e coeficientes de determinação $\left(R^{2}\right)$ dos três modelos que melhor se ajustaram aos dados observados de $\theta$, CEa e CEw, para o solo de $\mathrm{CT}_{\mathrm{f}}$, na estimativa da CEw, para concentrações de $\mathrm{K}$ na solução do solo, inferiores a $60 \mathrm{mg} \mathrm{L}^{-1}$

\begin{tabular}{|c|c|c|c|c|c|c|c|c|}
\hline \multirow{3}{*}{ Autor } & \multicolumn{7}{|c|}{ Solo de classe textural franca $\left(\mathrm{CT}_{\mathrm{f}}\right)$} & \multirow{3}{*}{$\mathrm{R}^{2}$} \\
\hline & \multirow{2}{*}{ D } & \multicolumn{6}{|c|}{$\begin{array}{ll}\text { Parâmetros } \\
\end{array}$} & \\
\hline & & a & b & C & d & CES & $\beta$ & \\
\hline Rhoades et al. (1976) & 0,9380 & 6,2464 & $-0,2887$ & - & - & 0,1232 & - & 0,7929 \\
\hline Vogeler et al. (1996) & 0,9331 & $-0,4310$ & $-0,2040$ & 2,7124 & 0,3368 & - & - & 0,7823 \\
\hline Mualen \& Friedman (1991) & 0,9190 & - & - & - & - & 0,0992 & 1,5417 & 0,7516 \\
\hline
\end{tabular}

(1996) foi o que melhor se ajustou aos dados observados na estimativa da concentração de $\mathrm{K}$ da solução do solo.

No que tange ao coeficiente angular, os ajustes dos modelos adaptados foram equâmines (em torno de 0,92), independentemente da relação entre CEw e K neles introduzida.

Com base no que se constatou na Tabela 6 e na Figura 4, para o solo de $\mathrm{CT}_{\mathrm{f}}$, procedeu-se a uma nova otimização dos ajustes dos modelos adaptados, considerando-se apenas os valores de $\theta, \mathrm{CEa}$ e $\mathrm{CEw}$, associados a concentrações de $\mathrm{K}$ na solução do solo, inferiores a $60 \mathrm{mg} \mathrm{L}^{-1}$. Os valores de $\mathrm{D}$, dos parâmetros e de $\mathrm{R}^{2}$ dessa nova otimização, explicitando CEw e K, são apresentados nas Tabelas 7 e 8, respectivamente.

Apesar dos modelos continuarem estimando bem a CEw, comparativamente aos ajustes obtidos e apresentados na Tabela 5 (solo de $\mathrm{CT}_{\mathrm{f}}$ ), verifica-se ligeira redução dos valores de $\mathrm{R}^{2}$ e de $\mathrm{D}$, quando se explicita CEw (Tabela 7); contudo, quando se explicita o K, substancial melhoria nos ajustes ( $\mathrm{R}^{2}$ passando de 0,60 para 0,80 e $\mathrm{D}$ de 0,85 para 0,94 ) dos modelos adaptados aos dados de concentração de $\mathrm{K}$ na solução do solo é evidenciada (Tabela 8), comparativamente aos obtidos e apresentados na Tabela 6 (solo de $\mathrm{CT}_{\mathrm{f}}$ ); observa-se, com isso, o quanto esses modelos são frágeis para estimar a concentração de $\mathrm{K}$ na solução do solo, quando esta concentração se situa acima de $60 \mathrm{mg} \mathrm{L}^{-1}$. Nota-se, também, que, para concentrações de K na solução do solo inferiores a $60 \mathrm{mg} \mathrm{L}^{-1}$, não há diferença expressiva dos ajustes dos modelos aos dados observados, independentemente do tipo de relação entre CEw e K, neles introduzida (Tabela 8).

Na Figura 6, o K estimado por meio dos modelos adaptados com uma relação entre CEw e $\mathrm{K}$ do tipo potência (A) e linear (B), para concentrações de K na solução do solo infe- 
Tabela 8. Coeficiente de concordância (D), parâmetros e coeficiente de determinação $\left(R^{2}\right)$ para o solo de $C_{f}$, na estimativa da concentração de $\mathrm{K}$ na solução do solo, para concentrações de $\mathrm{K}$ inferiores a $60 \mathrm{mg} \mathrm{L}^{-1}$

\begin{tabular}{|c|c|c|c|c|c|c|c|c|c|c|}
\hline \multirow{2}{*}{ Autor } & \multirow{2}{*}{ D } & \multicolumn{8}{|c|}{ Parâmetros } & \multirow{2}{*}{$\mathbf{R}^{2}$} \\
\hline & & a & b & C & d & CEs & $\beta$ & $\alpha$ & $\rho$ & \\
\hline $\begin{array}{l}\text { Rhoades et al. (1976) adaptado para a } \\
\text { relação CE-K do tipo potência }\end{array}$ & 0,9376 & 3,7011 & 1,0854 & - & - & 0,0457 & - & 0,0661 & 0,7217 & 0,7961 \\
\hline $\begin{array}{l}\text { Rhoades et al. (1976) adaptado para a } \\
\text { relação CE-K do tipo linear }\end{array}$ & 0,9370 & 0,8923 & 0,4041 & - & - & 0,0350 & - & 0,6106 & 0,0622 & 0,7946 \\
\hline $\begin{array}{l}\text { Vogeler et al. (1996) adaptado para a } \\
\text { relação CE-K do tipo potência }\end{array}$ & 0,9383 & $-0,1455$ & $-0,0839$ & 2,0490 & 0,1559 & - & - & 0,0862 & 0,7429 & 0,7972 \\
\hline $\begin{array}{l}\text { Vogeler et al. (1996) adaptado para a } \\
\text { relação CE-K do tipo linear }\end{array}$ & 0,9373 & 0,8705 & 0,0167 & 0,0729 & 0,0035 & - & - & $-4,7204$ & 0,6655 & 0,7950 \\
\hline $\begin{array}{l}\text { Mualen \& Friedman (1991) adaptado para } \\
\text { a relação CE-K do tipo potência }\end{array}$ & 0,9378 & - & - & - & - & 0,0489 & 1,4382 & 0,0864 & 0,7311 & 0,7964 \\
\hline $\begin{array}{l}\text { Mualen \& Friedman (1991) adaptado para } \\
\text { a relação CE-K do tipo linear }\end{array}$ & 0,9371 & - & - & - & - & 0,0398 & 1,3442 & 0,2140 & 0,0233 & 0,7948 \\
\hline
\end{tabular}

A.
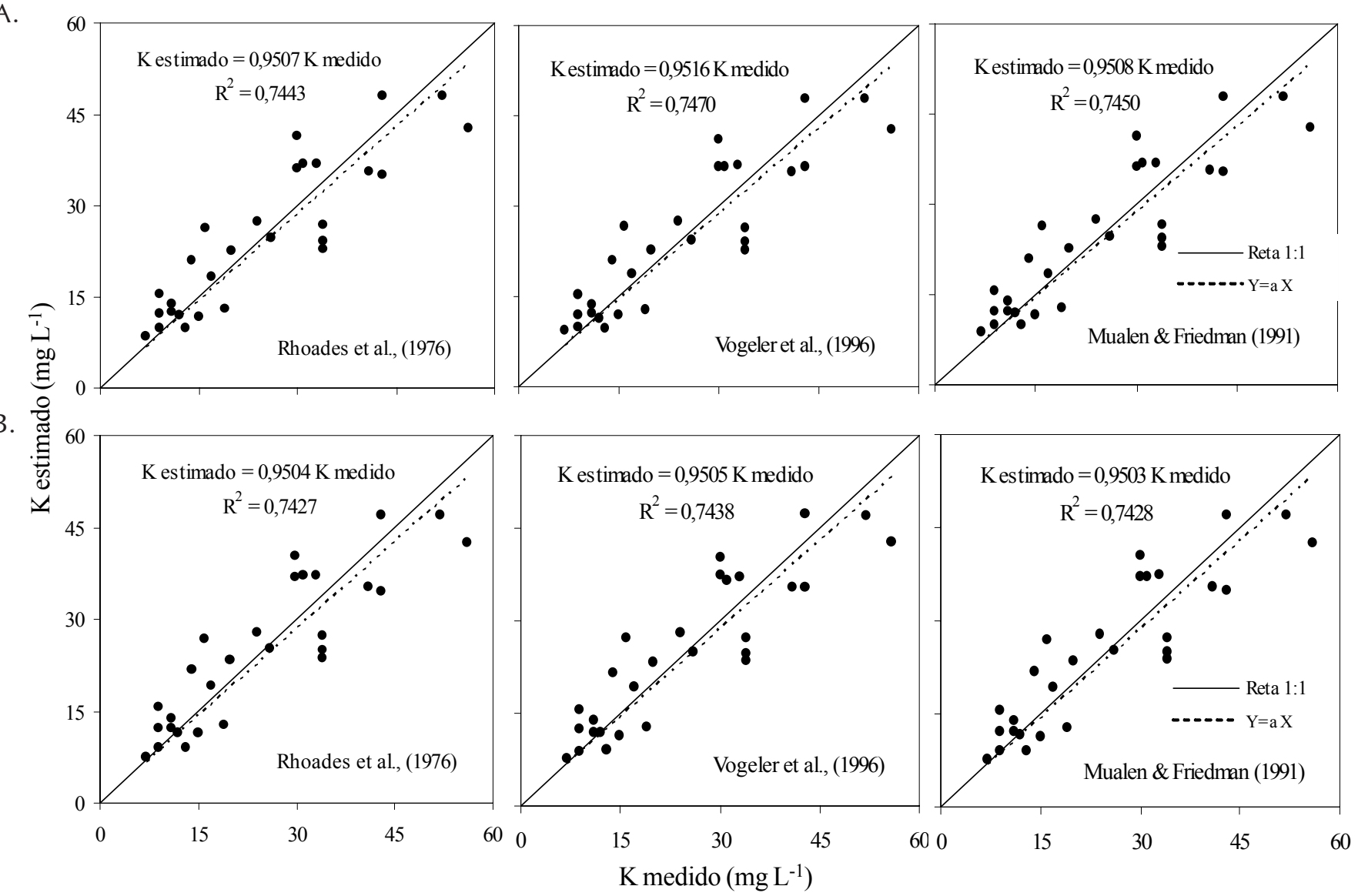

Figura 6. Relação entre o K medido e estimado para o solo de $\mathrm{CT}_{\mathrm{f}}$

riores a $60 \mathrm{mg} \mathrm{L}^{-1}$, é plotado como função do $\mathrm{K}$ medido por meio de uma equação do tipo $\mathrm{Y}=\mathrm{aX}$. Não se observa superioridade expressiva de nenhum dos modelos avaliados nem mesmo efeito do tipo de relação entre $\mathrm{CEw}$ e $\mathrm{K}$, neles inseri$\mathrm{da}$, significando que qualquer um dos modelos avaliados pode ser utilizado para se estimar a concentração de $\mathrm{K}$ na solução do solo, a partir de dados de $\theta$ e CEa obtidos pela técnica da TDR, independentemente do tipo de relação entre
CEw e K (potência ou linear) neles introduzida. Observa-se satisfatório ajuste da relação entre o $\mathrm{K}$ estimado e medido $\left(\mathrm{R}^{2}=0,75\right.$ e coeficiente angular superior a 0,95 ) (Figura $6 \mathrm{~A}$ e B). Ressalta-se que o fato de se considerar os modelos adaptados capazes de estimar a concentração de $\mathrm{K}$ na solução do solo, apenas na faixa de 0 a $60 \mathrm{mg} \mathrm{L}^{-1}$, para o solo de $\mathrm{CT}_{\mathrm{f}}$, além de conferir maior confiabilidade em suas estimativas conferiu, também, melhor ajuste entre dados estimados e 
medidos. Essas constatações reforçam a afirmação de que a textura do solo tem influência na estimativa da concentração de K na solução do solo, por esses modelos, tornando esses capazes de estimá-la para solos com características texturais iguais às do solo de $\mathrm{CT}_{\mathrm{f}}$, apenas quando esta concentração se situa abaixo de $60 \mathrm{mg} \mathrm{L}^{-1}$.

\section{CONCluSÃO}

É viável estimar a concentração de $\mathrm{K}$ na solução do solo, a partir de dados de $\theta$ e CEa, obtidos por meio da técnica da TDR, para condições de laboratório, por meio dos modelos de Rhoades et al. (1976), Vogeler et al. (1996) e Mualen \& Friedman (1991) adaptados com uma relação entre CEw e K do tipo potência, para as faixas de 0 a 60 e 0 a $120 \mathrm{mg} \mathrm{L}^{-1}$, para solos de textura franca e franco-arenosa, respectivamente.

\section{LITERATURA CITADA}

Bernardo, S. Manual de irrigação. 6.ed. Viçosa: UFV, 1995. $657 \mathrm{p}$.

Coelho, E.F.; Or, D. A parametric model for two-dimensional water uptake by corn roots under drip irrigation. Soil Science Society of America Journal, Madison, v.60, n.6, p.10391049, 1996.

Dalton, F.N.; Herkelrath, W.N.; Rawlins, D.S.; Rhoades, J.D. Time-domain reflectometry: Simultaneous measurement of soil water content and electrical conductivity with a single probe. Science, Washington, v.224, n.4651, p.989-990, 1984.

Folegatti, M.V. Fertirrigação: Citrus, flores e hortaliças. Guaíba: Livraria e Editora Agropecuária Ltda., 1999, 460p.

Giese, K.; Tiemann, R. Determination of the complex permitivity from the sample time domain reflectometry. Advanced Molex Relaxes Processes, New York, v.7, n.1, p.45-49, 1975.

Heimovaara, T.J.; Focke, A.G.; Bouten, W.; Verstranten, J.M. Assessing temporal variations in soil water composition with time domain reflectometry. Soil Science Society of America Journal, Madison, v.59, n.3, p.689-698, 1995.

Ledieu, J.; De Ridder, P.; De Clerck, P.; Dautrebande, S. A method measuring soil water moisture by time-domain reflectometry. Journal of Hydrology, Amsterdam, v.88, n.1, p.319$328,1986$.

Mmolawa, K.; Or, D. Root zone solute dynamics under drip irrigation: Review. Plant and Soil, Dordrecht, v.222, n.1-2, p.163-190, 2000.

Mualen, Y.; Friedman, S.P. Theoretical prediction of electrical conductivity in saturated and unsaturated soil. Water Resources Research, Washington, v.27, n.10, p.2771-2777, 1991.

Muñoz-Carpena, R.; Regalado, C.M.; Alvarez-Benedí, J.; Socorro, A.R.; Pérez, N. Determinación simultánea mediante TDR del transporte de agua y un soluto salino en el suelo. In: López, J.J.; Quemada, M. Temas de investigación en zona no saturada. 5.ed., Pamplona: Universidade Pública de Navarra, 2001, p.1-7.
Nadler, A. Discrepancies between soil solution concentration estimates obtained by TDR and aqueous extracts. Australian Journal Soil Research, Canberra, v.35, n.3, p.527-537, 1997.

Noborio, K. Measurement of soil water content and electrical conductivity by time domain reflectometry: A review. Computers and Electronics in Agriculture, Madison, v.31, n.3, p.213-237, 2001.

Persson, M.; Uvo, C.B. Estimating soil solution electrical conductivity from time domain reflectometry measurements using neural networks. Journal of Hydrology, Amsterdam, v.273, n.1-4, p.249-256, 2003.

Pinto, J.M. Fertirrigação em fruticultura irrigada. Revista Irrigação \& Tecnologia Moderna (ITEM). Associação Brasileira de Irrigação e Drenagem (ABID), v.49, n.1, p.14-23, 2001.

Rhoades, J.D.; Manteghi, N.A.; Shouse, P.J.; Alves, W.J. Soil electrical conductivity and salinity: New formulations and calibrations. Soil Science Society of America Journal, Madison, v.53, n.2, p.433-439, 1989.

Rhoades, J.D.; Raats, P.A.; Prather, R.J. Effects of liquid phase electrical conductivity, water content and surface conductivity on bulk soil electrical conductivity. Soil Science Society of America Journal, Madison, v.40, n.5, p.651-655, 1976.

Richards, L.A. (ed.). Diagnosis and improvement of saline and alkali soils. Washington: United States Salinity Laboratory, 1954. 160p. USDA Agriculture Handbook 60

Tommaselli, J.T.G.; Bacchi, O.O.S. Calibração de um equipamento de TDR para medida de umidade de solos. Pesquisa Agropecuária Brasileira, Brasília, v.36, n.9, p.1145-1154, 2001.

Topp, G.C.; Davis, J.L. Electromagnetic determination of soil water content: measurements in coaxial transmission lines. Water Resources Research, Washington, v.16, n.2, p.574$582,1980$.

Vogeler, I.; Clothier, B.E.; Green, S.R. TDR estimation of the concentration of electrolyte in the soil solution. Australian Journal Soil Research, Canberra, v.35, n.3, p.515-526, 1997.

Vogeler, I.; Clothier, B.E.; Green, S.R.; Scotter, D.R.; Tillman, R.W. Characterizing water and solute movement by TDR and disk permeametry. Soil Science Society of America Journal, Madison, v.60, n.1, p.5-12, 1996.

Ward, P.R.; Dunin, F.X. Growing season evapotranspiration from duplex soils in south-western Australia. Agricultural Water Management, Amsterdam, v.50, n.2, p.141-159, 2001.

Willmontt, C.J. On the validation of models. Physical Geography, New York, v.2, p.184-194, 1981.

Wraith, J. M; Das, B.S. Monitoring soil water and ionic solute distributions using time domain reflectometry. Soil and Tillage Research, Amsterdam, v.47, n.1-2, p.145-150, 1998.

R. Bras. Eng. Agríc. Ambiental, v.11, n.2, p.142-151, 2007. 